\title{
Investigation of optical and photoelectric properties of poly (o- hydroxyamide) sensitized by phthalocyanine as a perspective material for solar cells
}

\author{
Elvira Fazalova ${ }^{1} *$, Konstantin Kochunov ${ }^{1}$, Elena Bodyago ${ }^{1}$, Georgii Konoplev ${ }^{1}$, Nikolay Mukhin ${ }^{1}$, Irina Sokolova ${ }^{1}$, \\ Dmitrii Chigirev ${ }^{1}$, Andrey Ziminov ${ }^{2}$, Ludmila Rudaya $^{2}$, and Galina Lebedeva ${ }^{3}$ \\ ${ }^{1}$ Saint-Petersburg Electrotechnical University, 197376, Saint-Petersburg, Russia \\ ${ }^{2}$ Saint-Petersburg State Institute of Technology (Technical University), 190013, Saint-Petersburg, Russia \\ ${ }^{3}$ Institute of Macromolecular Compounds RAS, 199004, Saint-Petersburg, Russia
}

\begin{abstract}
Optical and photoelectric properties of poly (o- hydroxyamide) (PHA) sensitized with zinc phthalocyanines were investigated in the visible and near infrared spectral regions. The structures were deposited on glass substrates by centrifugation and subsequent drying of a PHA film without thermal annealing. Optical spectra revealed characteristic absorption peaks of phthalocyanine in the longer wavelength region at $620-640 \mathrm{~nm}$ and $680-700 \mathrm{~nm}$; absorption of the PHA matrix monotonously increases to the shorter wavelengths starting from $700 \mathrm{~nm}$. Measurements of the photocurrent under irradiation with a high-power LED at a wavelength $630 \mathrm{~nm}$ showed photoconductivity related to the organic dye; photoconductivity also was observed while irradiated at $540 \mathrm{~nm}$, presumably due to the absorption of PHA matrix. For non-sensitized (dye-free) PHA films no detectable photocurrents were produced by $630 \mathrm{~nm}$ irradiation. It was shown that introducing of phthalocyanines significantly improves optical absorption and photoconductivity of PHA thin films at the wavelengths, where the maximum in the spectral distribution of solar radiation lies. It was concluded that phthalocyanine-sensitized PHA has the potential use as a photosensitive organic material for solar applications, for example in developing composite organicinorganic structures with ferroelectrics.
\end{abstract}

\section{Introduction}

High initial costs of silicon based solar panels are one of the main factors hampering widespread use of solar energy, especially in pure countries. More affordable organic and organic-inorganic composite photosensitive materials potentially suitable for development of cheap solar cells are now under extensive investigation worldwide.

Dye-sensitized solar cells (DSSC) mainly use ruthenium complex dyes (N3 and N719) as sensitizer due to their broad optical absorption spectrum. However, the relatively low molar extinction coefficients of dyes based on them and their high synthesis cost limit their further use in DSSC [1]. Therefore, the introduction of sensitizing dyes with higher molar extinction coefficients in DSSCs is an important alternative method for the manufacture of solar cells.

In search of a replacement for ruthenium compounds, a variety of organic synthetic materials are being investigated as an inexpensive and effective dye. Recent years have shown that scientists have achieved good results in converting sunlight into energy, using natural dyes. Among the most promising materials are pentacenes (Pn) and metal phthalocyanines (MPc).
Phthalocyanines have a great advantage with respect to the mobility of charge carriers and photo-properties by changing the modification of molecular structures with various central metals and chemical substitutions [2, 3].

Among metal-phthalocyanines, zinc phthalocyanine $(\mathrm{ZnPc})$ is widely used as ahole transport material. In their work, Hong et al. reported an energy conversion efficiency about $2.2 \%$ under illuminated with AM1.5 in a heterojunction $(\mathrm{ZnPc}) / \mathrm{C}_{60}$ thinfilmcell [4].

The effect of chenodeoxycholic acid (co-adsorbent) on a $\mathrm{TiO}_{2}$ sensitized with $\mathrm{ZnPc}$ is noted in the research[5]; the highest efficiency $2.93 \%$ was achievedwiththe acid content of $1 \mathrm{mM}$.

The aim of this work is to study the optical and photoelectric properties of poly (o-hydroxyamide) (PHA) sensitized with zinc phthalocyanine in the visible and near infrared regions as a prospective material for solar energy applications.

\section{Formation of the PHA/phthalocyanine thin film structures}

The synthesis of zinc (II) 2,9,17,23-tetrakis(4-(3,5bis(trifluoromethyl)-1H-pyrazol-1-

yl)phenoxy)phthalocyanine ( $\mathrm{ZnPc})$ was carried out by

*Corresponding author: fazalova.el@gmail.com 
the method described elsewhere [6]. The polymer matrix material was poly (o-- hydroxyamide) (PHA) solution, the kinematic viscosity of the initial solution was $v=640$ cSt, the dry polymer concentration was $20 \%$. The concentration of $\mathrm{ZnPc}$ was varied in the range $1.83-3.7 \%$ by weight of dry polymer.

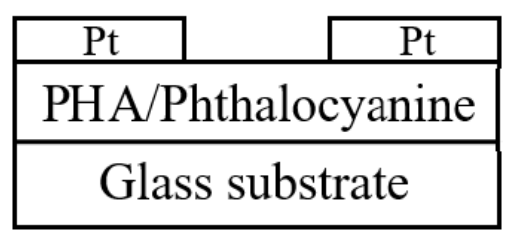

Fig. 1.Thin film PHA/phthalocyanine photoconductive structure.

Phthalocyanine-sensitized polymer films were deposited on glass substrates (Fig. 1) by centrifugation at various centrifuge speeds from $1500 \mathrm{rpm}$ to $2000 \mathrm{rpm}$ for 30 seconds. After centrifugation samples were dried for 40 minutes without any thermal annealing. The upper Pt electrodes with a gap of $500 \mu \mathrm{m}$ for subsequent photoelectric measurements were applied through a shadow mask by the method of ion-plasma sputtering.

Overall, 11 samples with different dye concentrations and thicknesses were fabricated (Table 1), for the article we selected samples on which the maximum photocurrent was observed.

The interference patterns in the optical transmission spectra were used to estimate the thicknesses of the films. Spectra were recorded using AvaSpec-2048 fibreoptic CCD spectrophotometer in the wavelength range 400-1100 nm, the thicknesses were estimated with AvaSoft-ThinFilm software.

Table 1.Test samples.

\begin{tabular}{|c|c|c|c|}
\hline Sample & Description & $\begin{array}{l}\omega, \\
\text { r/min }\end{array}$ & Thickness, $\mu \mathrm{m}$ \\
\hline$\# 1$ & \multirow{2}{*}{ PHA $20 \%$} & 1500 & 3.3 \\
\hline$\# 2$ & & 2000 & 2.3 \\
\hline$\# 3$ & \multirow{3}{*}{ PHA + Dye $1.87 \%$} & \multirow{3}{*}{1500} & 4.7 \\
\hline$\# 4$ & & & 4.7 \\
\hline$\# 5$ & & & 4.7 \\
\hline$\# 6$ & \multirow{2}{*}{ PHA + Dye $1.87 \%$} & \multirow{2}{*}{2000} & 2.3 \\
\hline$\# 7$ & & & 2.3 \\
\hline$\# 8$ & \multirow{4}{*}{ PHA + Dye $3.7 \%$} & \multirow{2}{*}{1500} & 2.3 \\
\hline$\# 9$ & & & 2.3 \\
\hline$\# 10$ & & \multirow{2}{*}{2000} & 1.7 \\
\hline$\# 11$ & & & 1.5 \\
\hline
\end{tabular}

\section{Investigation of the optical properties of PHA/phthalocyanine}

The reflection $R(\lambda)$ and transmission $T(\lambda)$ spectra of the PHA/Phthalocyanine test samples were recorded in the 400-1000 nm wavelength range with AvaSpec-2048 spectrophotometer; for measuring reflection spectra a special fibre-optic probe was employed, an aluminized mirror with a reflectivity about $95 \%$ was used as a reference. The absorption spectra of the samples were calculated from reflection and transmission using Beer-
Lambert equation. Spectral measurements were carried out at room temperature.

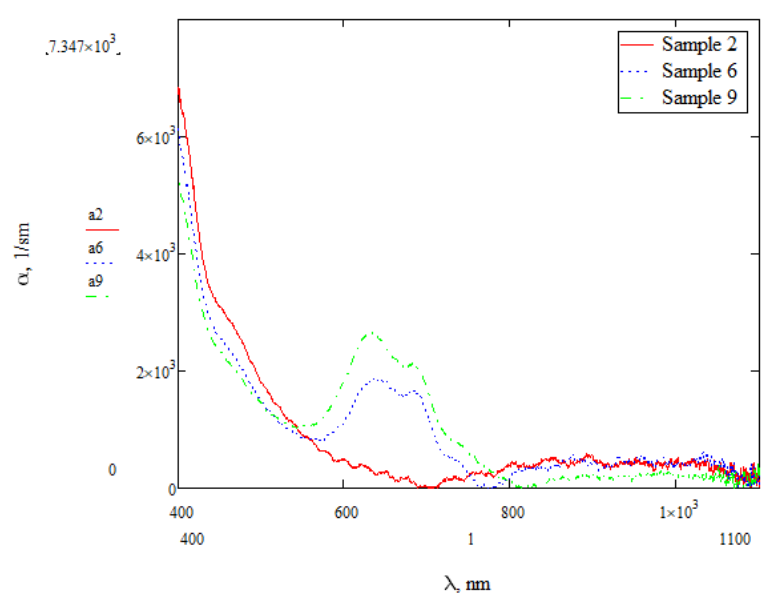

Fig. 2.Spectral dependence of the absorption coefficient for samples with different dye concentrations and the same thickness.

Figure 2 shows the spectral dependence of the absorption coefficient for three samples of the same thickness of $2.3 \mu \mathrm{m}$, but with different concentrations of phthalocyanine dye (samples \#2, \#6 and \#9 in Table 1). It can be seen from Figure 2 that sensibilization with phthalocyanine dye leads to the appearance of two additional absorption bands in the spectra at the wavelengths 620-640 $\mathrm{nm}$ and 680-700 $\mathrm{nm}$ in comparison with non-sensitized material(dye-free PHA polymer matrix). Absorption of the polymer matrix monotonously increases to the shorter wavelengths starting from 700 $\mathrm{nm}$; presumably, PHA has a strong absorption band in the UV region [7]. In the longer wavelengths part of the spectra starting from $800 \mathrm{~nm}$ both PHA matrix and phthalocyanine are virtually transparent. According to the absorption spectra, it can be concluded that an increase in the concentration of the dye in the initial solution leads to an increase in absorption at the wavelength range of $600-800 \mathrm{~nm}$. With an increase of the phthalocyanine dye concentration, the absorptivity of the developed structures improves. Moreover, the absorption maxima of the dye are close to the maximum in the spectral distribution of solar radiation, which potentially makes it a suitable photosensitive material for solar cells.

It is known from literature that phthalocyanines are characterized by the presence of a Soret band in the UVvisible spectrum in the region of 300-400 $\mathrm{nm}$ and an intense Q-band in the region of 600-750 nm [8]. The Qband is attributed to the photoexcitation of individual phthalocyanine molecules on the grounds that they all have the same aromatic macrocycle, which includes nitrogen atoms and contains 18 conjugated $\pi$-electrons [9].

The long-wavelength maxima in the spectra in Figure 2 , as we assume, are associated with the Q-absorption band of phthalocyanine, since such maxima are not observed for the sample with zero dye concentration. The existence absorption peak at $630 \mathrm{~nm}$ clear indicated that zinc phthalocyanine is aggregated in polymer films 
[10]. In agreement with literature [11, 12], the peak in the Q-band, corresponding to the phthalocyanine monomer species $(680 \mathrm{~nm})$ is slightly shifted to $671 \mathrm{~nm}$.

Moreover, the position and shape of the peaks can vary from the peripheral substituents as well as from the properties of the structures themselves [13, 14].As mentioned earlier, peripheral substituents can affect the shape of the peaks, for example, [15] zincphthalocyanine dyes with a noticeable difference in the molecular structure were used. They attributed a significant difference in the ability of various dyes to generate a photocurrent with the difference in substituents attached to the mainphthalocyaninecore.

It should be noted that two almost equally intense and wide absorptions bands at the wavelengths $620-640$ $\mathrm{nm}$ and $680-700 \mathrm{~nm}$, which are characteristic for the $\mathrm{ZnPc}$ dye used in this research or in the paper [16], provide better utilizing of solar radiation comparing with phthalocyanines with only one absorption band, for example in the work [17].

\section{Investigation of photoelectric properties of PHA/phthalocyanine}

Photoconductivity of the test samples were measured with Keithley 6482 double-channel picoammeter/voltage source. The samples were irradiated with ultra-bright LEDs at the wavelengths $540 \mathrm{~nm}$ (outside phthalocyanine absorption bands) and $630 \mathrm{~nm}$ (inside phthalocyanine absorption bands). The LEDs were located at a distance of $10 \mathrm{~mm}$ from the samples and powered with rectangular voltage pulses with 20 seconds duration, the operating currents of both LEDs were adjusted in such a way as to produce equal photon flux density upon the surface of the sample at different wavelengths. Photoelectric measurements were carried out at room temperature; results are presented for the samples with the highest photocurrent observed.

Figures 3 and 4 shows the time dependences of the transient currents at a constant bias voltage of $5 \mathrm{~V}$ and pulsed irradiation at a wavelength of $540 \mathrm{~nm}$ fora nonsensitized sample. At a wavelength of $540 \mathrm{~nm}$, photosensitivity is related to the absorption in PHA matrix (the photocurrent is about $5 \mathrm{pA}$ ), while at a wavelength of $630 \mathrm{~nm}$ the photocurrent is not detectable.

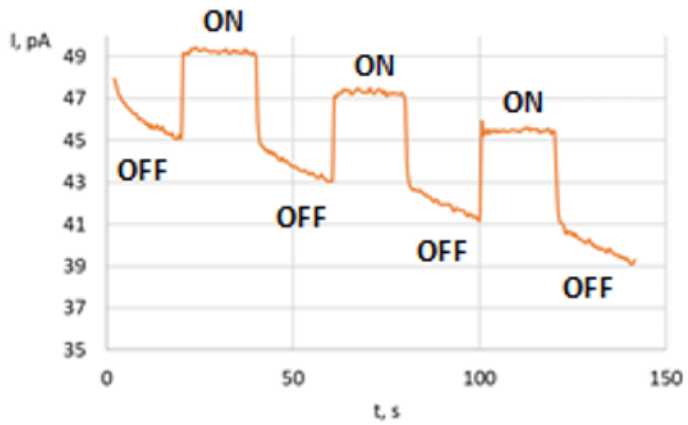

Fig. 3.Transient current under pulsed irradiation at $540 \mathrm{~nm}$ for the sample \#2 (non-sensitized). "ON" - LED is turned on, "OFF" - LED is switched off.

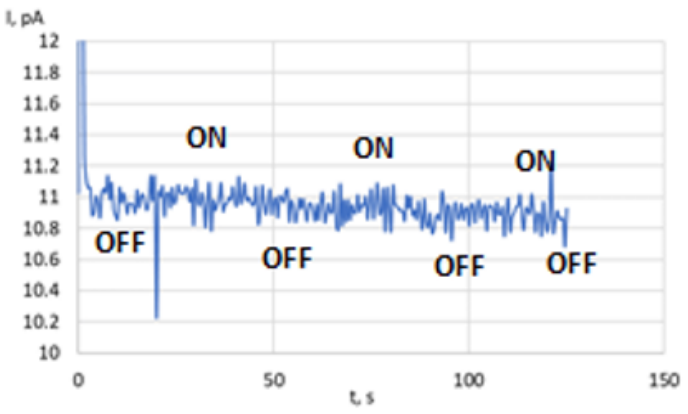

Fig. 4.Transient current under pulsed irradiation at $630 \mathrm{~nm}$ for the sample \#2 (non-sensitized).

Figures 5 and 6 shows the time dependences of the transient currents for a sensitized sample with the concentration of phthalocyanine $1.87 \%$. When irradiated at $540 \mathrm{~nm}$ the character of photoconductivity is very similar to a non-sensitized sample, because optical absorption at this wavelength is determined by PHA matrix. On contrary, prominent photocurrents can be observed with $630 \mathrm{~nm}$ irradiation due to phthalocyanine absorption.

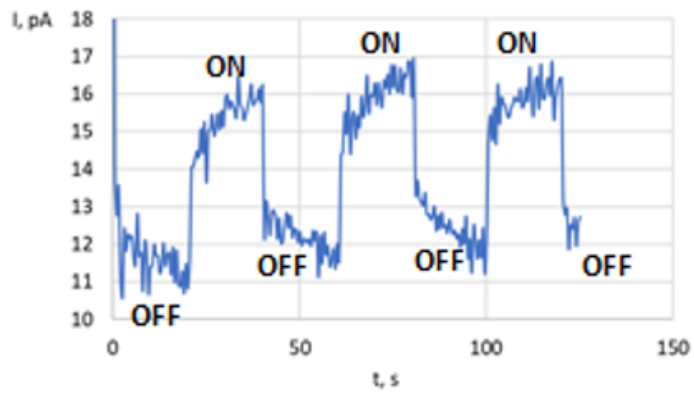

Fig. 5.Transient current under irradiation at $540 \mathrm{~nm}$ for the sample \#6 (sensitized with phthalocyanine).

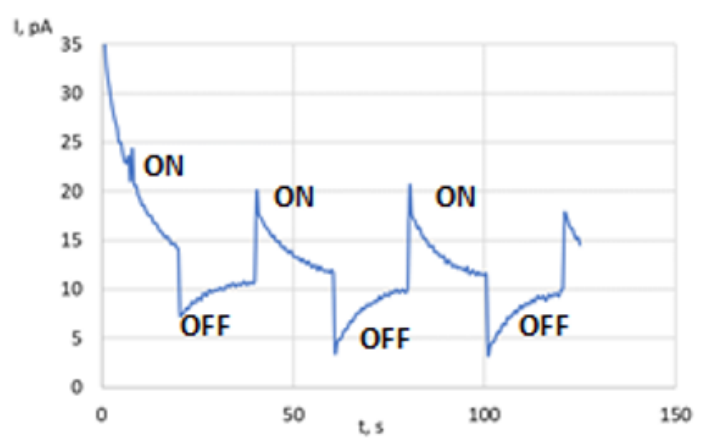

Fig. 6. Transient current under irradiation at $630 \mathrm{~nm}$ for the sample \#6 (sensitized with phthalocyanine).

Absolute values of the photocurrent in the PHA/ZnPc system are quite low, which could be explained by pure conductivity of PHA. We assume that photoelectric properties of this system could be improved by matrix modification.

\section{Conclusion}

In this work, a set of thin films synthesized on glass substrate from a poly (o-hydroxyamide) (PHA) solution 
sensitized with different concentrations of zinc phthalocyanine, centrifuged, and then dried for 40 minutes without thermal annealing were investigated. The following conclusions could be made from analysis of the optical absorption in the wavelength range of 400$1000 \mathrm{~nm}$ : the spectra of the films, deposited at different centrifuge rotational speeds, differ insignificantly; greater differences are noticeable for structures with different dye concentrations; the absorption spectra show peaks in the short-wavelength and long-wavelength parts of the spectrum. It was suggested that in the shortwavelength part, absorption is related the PHA matrix, while in the long-wavelength part it is produced by phthalocyanine. It was shown that sensitization with a phthalocyanine dye leads to the appearance of $Q$ absorption bands in the long-wavelength part of the spectrum.

Measurements of the photocurrents under irradiation with a high power LED at $630 \mathrm{~nm}$ which corresponds to the phthalocyanine absorption peak showed photoconductivity related to the organic dye; the photocurrent also was observed while irradiated at 540 nm, presumably due to absorption in PHA matrix. For non-sensitized PHA films there were no detectable photocurrents with $630 \mathrm{~nm}$ irradiation.

Thus, introducing of phthalocyanines significantly improves optical absorption and photoconductivity of poly (o- hydroxyamide) organic thin films in the wavelength region, where the maximum in the spectral distribution of solar radiation lies. It can be concluded that phthalocyanine-sensitized poly (o- hydroxyamide) has the potential use as a photosensitive organic material for solar applications, for example in developing composite organic-inorganic structures with ferroelectrics.

\section{Acknowledgements}

This research was conducted in the framework of the project №0788-2020-0008, carried out within the framework of the state assignment № 075-00306-20-01 Ministry of Science and Higher Education of the Russian Federation.

\section{References}

[1] J. He, B. Wang, S. Chang, T. Chen, Org.-metal. and Rel. Molec. forEner. Conv., 23 (2015)

[2] D. Wöhrle, G. Schnurpfeil, S.G. Makarov, A. Kazarin, O.N. Suvorova, Macroheterocycles 5, 191 (2012)

[3] N. Kaur, M. Singh, D. Pathak, T. Wagner, J.M. Nunzi, Synth. Met., 190, 20-26 (2014)

[4] Z.R. Hong, B. Maennig, R. Lessmann, M. Pfeiffer, K. Leo, Appl. Phys. Lett., 90 (2007)

[5] J.H. Yum, S.R. Jang, R. Humphry-Baker, M. Gratzel, J.J. Cid, T. Torres, Md.K. Nazeeruddin, Langmuir, 24, 5636-5640 (2008)
[6] A.V. Ziminov, M.A. Furman, A.I. Kolganova, S.M. Ramsh, Russ. J. Org. Chem., 52, 133-135 (2016)

[7] A.E. Bursian, N.V. Klimova, L.I. Rudaya, I.M. Sokolova, V.A. Lukoshkin, G.K. Lebedeva, Phys. Solis State, 50, 557-561 (2008)

[8] Z.U. Islam, M. Tahir, W.A. Syed, F. Aziz, F. Wahab, S.M. Said, M. Sarker, S.H. Md Ali, M.F. MohdSabri, Energies, 13, 962 (2020)

[9] J. Simon, J.-J. Andre, Springer Verlag 13 (1985)

[10] A.V. Ziminov, M.A. Furman, E.V. Borisov, N.S. Seravin, S.M. Ramsh, Macroheterocycles, 9, 387-394 (2016)

[11] M.C. Larciprete, R. Ostuni, A. Belardini, M. Alonzo, G. Leahu, E. Fazio, C. Sibilia, M. Berlotti, Photonics Nanostruct, 5, 73-78 (2007)

[12] S. Subbiah, R. Mokaya, J. Phys. Chem. B. 109, 5079-5084 (2005)

[13] V.L. Berkovits, A.V. Ziminov, A.G. Kazanskiı̌, A.G. Kolos'ko, S.M. Ramsh, E.I. Terukov, A.V. Fenukhin, V.P. Ulin, T.A. Yurre, J.P. Kleider, Phys. Solid State, 49, 272-277 (2007)

[14] A.G. Kazanskii, E.I. Terukov, A.V. Ziminov, O.B. Gusev, A.V. Fenukhin, A.G. Kolosko, I.N. Trapeznikova, Yu.A. Nikolaev, B. Modu. Tech. Phys. Lett. 31, $782-784$ (2005)

[15] D. Wrobel, A. Boguta, J. Photochem. Photobiol. C., 150, 67-76 (2002)

[16]Z.U. Islam, M. Tahir, W.A. Syed, F. Aziz, F. Wahab, S.M. Said, M.R. Sarker, S.H.Md. Ali, Sabri. Energies, 13, 962 (2020)

[17] M.M. Stylianakis, D. Konios, G. Viskadouros, D. Vernardou, N. Katsarakis, E. Koudoumas, S.H. Anastasiadis, E. Stratakis, E. Kymakis, Dyes and Pigments, 146, 408-413 (2017) 